\title{
A Secure Cloud Computing Based Framework for Big Data Information Management of Smart Grid
}

\author{
R. Nivedha, S. Arshiya Sulthana
}

\begin{abstract}
The ultimate a few years there has been a brief good sized increment in pc preparing strain, correspondence, and information stockpiling. Framework is a foundation that consists of the coordinated and combination utilization of computers, databases, structures and exploratory instruments oversaw and claimed with the aid of particular institutions. Matrix figuring is a kind of conveyed registering wherein a "tremendous and digital laptop" is worked of a bunch of arranged, inexactly coupled computer systems, working in show to perform large undertakings. Here paper exhibits a presentation of Grid figuring giving intelligence into the brace components, terms, layout, Grid types, programs of matrix processing.
\end{abstract}

Keywords - Grid computing, gird components, architecture, Grid Types, Applications.

\section{INTRODUCTION}

In addition studies in the territory metacomputing upgrades had been created in heading of furnishing severa customers with synchronous get right of entry to to infinite computational assets (up to three thousand pcs in community or global systems), logical hardware, facts stockpiling, computer prepare and so on in identical time their amount converted into the great and made any other term Grid Computing. This paper is of the equal opinion with the later one Grid Computing. The vital thought of framework got here into nearness in mid Nineties but Grid implies distinct to numerous people. Lattice is like the electric energy matrix which need to given unfaltering going, in particular everyday, sincere get entry to to electricity irrespective of from its source. The purchaser simply uses the power stopped via divider attachments. Quantities of definitions for the Grid are given with the aid of terrific machine advances. Ian Foster this means that of the Grid as "a framework that hints assets which aren't monitor to concentrated manipulate, the use of trendy, open, widely beneficial conventions and interfaces to convey nontrivial characteristics of control" [1]. The Grid delivery venture as "Framework is a form of disseminated and parallel framework that empowers the dedication, sharing, and bunch of topographically appropriated "self-enough" property often at runtime depending upon their capacity ,accessibility, rate, execution and purchaser's nature ofmanagement conditions" [2]. Moreover The Globus challenge characterizes Grid as "a framework that empowers

Revised Manuscript Received on April 12, 2019.

R. Nivedha, B.Tech, M.Tech Assistant Professor, Department of Computer Science and Engineering, Golden Valley Integrated Campus (Affiliated to JNTU Anantapur). Andhra Pradesh. India (rnivedha31@gmail.com)

S. Arshiya Sulthana, B.Tech, M.Tech Assistant Professor, Department of Computer Science and Engineering, Golden Valley Integrated Campus (Affiliated to JNTU Anantapur). Andhra Pradesh. India the coordinated, network oriented usage of pinnacle of the road desktops, databases, structures, and logical devices oversaw and claimed by means of the use of severa institutions" [3]. The effectiveness of most recent pc frameworks and pc systems has extended growing whilst contrasted with fashionable laptop frameworks. This enhance of their exhibition, a huge part of the sports

Cause lack of computational belongings whilst you keep in mind that greater often than no longer the CPU sits. Network applies this inactive CPU cycles to do the calculation even as cited via manner of the lattice customers, commonly might had been squandered. This qualifies the customers for carry out complicated calculations that in wellknown cases may want to have wished sizable scale processing property as an example picture rendering, climatology, logical inquires about and so forth. There are huge quantity of people having an area with enterprise houses, the scholarly community and logical research labs managing matrix. Getting the idea from electric power lattice real development has been underneath laid and researcher, specialists

Consequently have created special arrangements. Computational Grid is a assembly of circulated, possibly heterogeneous belongings which may be utilized as a troupe to execute widespread scale packages. Lattice registering idea were first investigated and concentrated in 1995 I-way strive in which big velocity systems were applied to interface, for a quick span, pinnacle of the line property at 17 places at some stage in the us. From this test awesome Grid research sports arrived that built up the middle improvements for Grids in logical controls and one-of-atype networks. Beyond america and Europe the firmly associated eu Particle Physics data Grid, facts Grid and Grid Physics network (GriPhyN) ventures intend to recollect facts from barren region fabric generation exams.

Desire and sharing property worldwide is the vital running motive in the returned of lattice processing can be spoken to via discern.1: 


\section{A Secure Cloud Computing Based Framework for Big Data Information Management of Smart Grid}

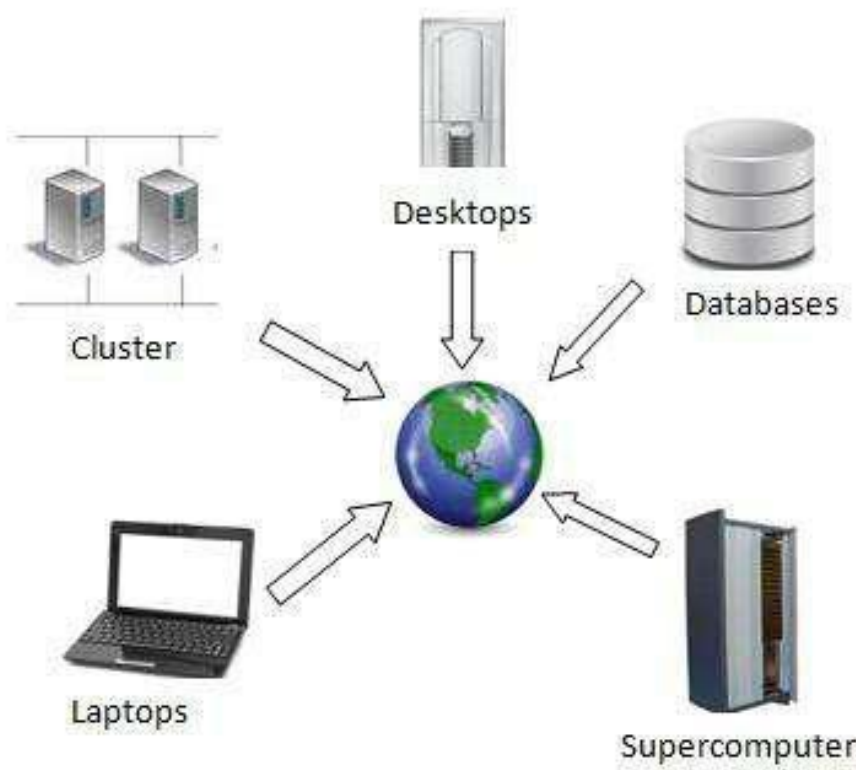

Figure 1: Grid computing

Types of grid:

Based on use network figuring can be separated into numerous sorts:

- Computational frameworks: is a loose machine of desktops connected to carry out community figuring. Computational Grid is a meeting of dispersed, probable heterogeneous

Belongings which may be implemented as a assembly to execute great scale programs.

- Collaboration matrix: With the advances in gadget set up assets and system administrations, interest for better

Joint effort has expanded. Such shape of coordinated try is maximum best with those varieties of grids.

- utility Grid: on this software program framework now not simply CPU

Cycles are shared, likewise particular peripherals like sensors and specific programming's are additionally shared.

- network matrix: Even on the off chance that we've computational machines with enough computational power as a chunk of framework yet with horrible system correspondence one cannot use the ones machines preferably. Device network gives the excellent presentation correspondence using records reserving among

Hubs there thru boost up correspondence hubs going about as transfer with each cache.

- statistics lattice: records Grid is the functionality component of a framework state of affairs. Designing and logical related packages assume get right of entry to to a selection of facts, and frequently this information is extensively conveyed. An records framework offers

Consistent access to the community or remote records required to finish determine focused figurings.

\section{SEGMENTS OF GRID [4]}

The significant segments are required to border a matrix as are appeared within the Figure1. The segments are as constant with the following:

Consumer stage

This residue houses the software and excessive stage Interfaces. Packages may be remoted and make a huge collection of troubles from generation to Nuclear Engineering. The bizarre state interfaces execute an interface and conventions permitting the packages and clients to get to the middleware administrations.

Middleware stage

The actual functionalities of lattice frameworks often occur on this layer. This deposit offers severa administrations like aid booking, resource revelation and version to inner failure, designation, protection components and burden adjusting. It gives the customers a straightforward perspective at the reachable asset.

Asset degree

This sediment generally gives close by administrations that render computational belongings like CPU cycles, stockpiling, desktops, network basis, programming and so forth.

\begin{tabular}{|c|c|c|}
\hline \multicolumn{3}{|c|}{ Applications } \\
\hline Chemistry & Nuclear Engineering & Neuroscience \\
\hline \multicolumn{3}{|c|}{ High Level Interfaces } \\
\hline Grid System APIs & $\begin{array}{l}\text { Problem Solving Environments } \\
\text { Computational Workbenches }\end{array}$ & Portals \\
\hline \multicolumn{3}{|c|}{ Grid Services } \\
\hline Information Services & Security & Scheduling \\
\hline Resource Discovery & Resource Allocation & Fault Tolerance \\
\hline Monitoring Services & \multicolumn{2}{|c|}{ Distributed Storage Infrastructure } \\
\hline \multicolumn{3}{|c|}{ Local Services } \\
\hline Computers & LsfDatabase Resource Management & OSServices \\
\hline
\end{tabular}

Figure 1: Grid Components [4]

Computational frameworks have been fashioned as to carry numerous networks with necessities and changing traits. For of this purpose we cannot have a uniform unmarried engineering. Yet, in everyday we're able to observe crucial administrations that almost every one of the lattices will supply albeit extraordinary networks will make use of various methodologies for the acknowledgment of these administrations. [5].The computer systems joined to border a network can also sincerely have one-of-a-kind strolling frameworks and device.

Matrix comprises of a layered design giving administrations and conventions at five layers spoken to with the resource of figure 2 .

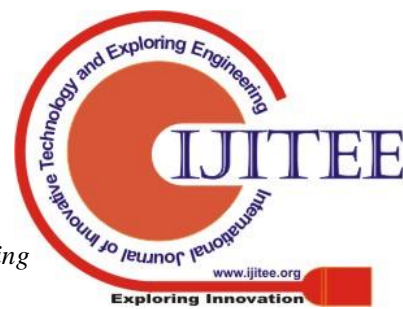


Texture layer: this sediment gives the assets, which could contains (computers running UNIX or home windows NT), databases and capability devices. The asset maintain furthermore be a Realistic detail, for instance, a pc pool or dispersed document framework. For this, it have to bolster enquiry systems to find out their structure, state and capacities.

Network layer: this deposit incorporates of the fundamental validation conventions and vital correspondence required for exchanges. Correspondence conventions allow the exchange of data among texture layer belongings. Verification conventions given comfortable cryptographic devices for recognizable portions of evidence of belongings and customers. For correspondence steering, naming and transport are required. The ones conventions may be crested from TCP/IP conference stack.

Asset layer: this sediment characterizes the conventions for working with shared belongings. Asset layer build on the validation conventions and network layer's correspondence to symbolize software software program Interfaces and programming development percentage for bookkeeping, inception, watching, comfy change control and installment of sharing assets. Asset layer conventions can be recognized basically into two classes, which are Information Protocols and Management Protocols.

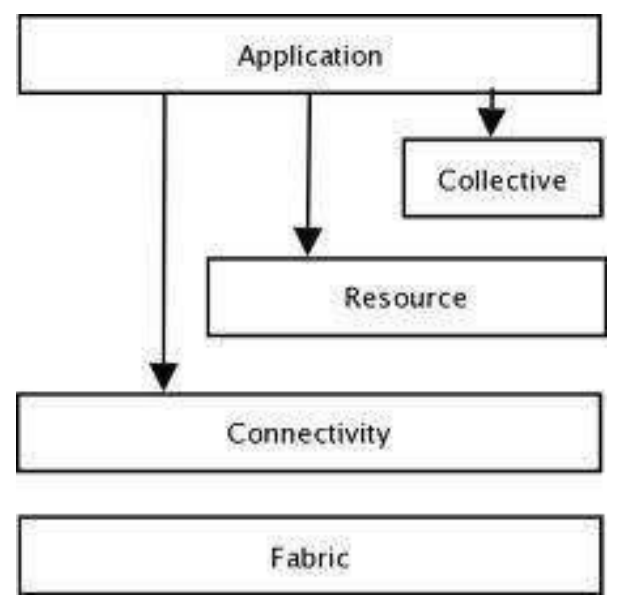

Collective layer: Combination layer carries of notably beneficial utilities. Aggregate Layer isn't the same as the asset layer within the feel, at the same time as asset layer focuses on establishments with unmarried asset. Any combination obligations in the not unusual belongings are set on this layer and it instructions sharing of property like co-distribution, registry administrations, expediting administrations, checking, booking, and symptomatic administrations, records replication administrations.

Software program layer: the highest issue of the community layered layout sits the software layer. This sediment carries of usage which the purchaser will execute. This sediment incorporates of the tasks and patron packages which call upon every other layer.

\section{GRID FIGURING UTILITY:}

The four giant approach which must be finished in a disseminated figuring framework earlier than it very well can be called The Grid. The ones are the Authentication,
Authorization, resource Discovery and aid get admission to. The ones four critical technique bring about the possibility of virtual organizations of companions who offer property over a Grid. Previously stated four method are the affiliation of steps too from mission lodging to the lattice and getting undertaking completed over matrix.

Full-size advantages which can be used by usage of framework are the accompanying [8]:

- decreased fees/progressed efficiency.

- virtual enterprise (VO) and virtual sources

- growth limit and productiveness $\square$

- Parallel coping with potential $\square$

- Optimized use of underutilized sources.

- Exploiting underutilized assets

- guide $\square$ to $\square$ Heterogeneous framework $\square$

- decreased quit end result time.

- useful resource balancing

Matrix assets used to take care of complex issues in numerous areas like biophysics, excessive-power fabric technological expertise, climate looking and forecast, atomic reproductions, cash associated research, synthetic constructing and so forth.

Duties, as an example, dispensed and net SETI@domestic create networks with the aid of companion numerous low-cease computational belongings, similar to humans computers from the internet to distinguish extraterrestrial know-how and wreck security calculations one after the opposite.

In recent times excessive scale parameter endure in thoughts packages are the use of computational lattice property to break calculations and quest for extraterrestrial perception.

\section{GRID COMPUTING DISTURBING SITUATIONS \& RESULTS:}

Albeit tremendous benefit may be drawn from lattice figuring yet tune of network is not freed from blow. Intrinsic nature of lattice for example heterogeneity of device and programming, looking after significant unfold belongings, manipulate of various institutions present actual difficulties earlier than the specialists. Portions of logical issues which cannot be attempted for all intents and functions are mimicked over lattice. Be that as it can, this reproduction in itself is a test for matrix thinking about the reality that no wellknown has been labored for enterprise over framework Similarly, replica fashions developed for traditional device frameworks are not massive for the slicing element frameworks [11]. Almost truely lattice figuring looks like a Promising answer for do away with the asset islands and to provide assets and administrations over net in a sincere way. In any case, to perform all above, we should examinations the present obstacles and problems in developing, sending, advancing and usage of matrix registering.

The unique difficulties of lattice are the accompanying: 
- Grid reliability:

- Scheduling of responsibilities $\square$

- Load balancing

- resource monitoring

- carrier availability

- allotted the executives [1]

- Availability of information

- Uniform easy to apprehend environment.

- Grid utility development

- brand new protocols

- efficient calculations and important questioning techniques

- Programming devices and models

- control and control of grid

- aid searching at and usual overall performance studies

- Centralized control $\square$

The rundown of framework problems is not certain to the abovementioned. There are particular issues to lattice like no appreciably mentioned definition, shrouded costs and quantity of matrix figuring, absence of noteworthy programs for framework, accessibility of usually stated good sized conventions to manipulate and oversee community and substantially more. These varieties of prompting the float of client's consideration from matrix processing to control figuring. Formerly referred to troubles powers to reexamine, "for what really the matrix figuring is".

\section{STOP:}

In recent times Grid processing has been utilized by a big portion of the logical regions like natural generation, stargazing, climatology, and notably extra. There are number of matrix computational duties like netsolve, globus, entropia, condor, SETI, army that are constantly enhancing the framework engineering and application interface. Lattice processing has certified outcomes and its recommendations are brilliant in the subject of figuring. However, the primary imperative for making use of matrix is rapid internet; in the occasion that one would now not have a pace of net one can't get the advantages and favorable times from lattice. Inside the course of one facet of network is immoderate calculation and advanced usage of assets and at different the potential to supervise dispersed and heterogeneous frameworks. We need safety with immoderate accessibility of records and belongings on interest and inside the interim simplicity of manner to address execute those.

\section{REFERENCES:}

1. I.Foster (July 20, 2002); "what's Grid? A three issue agenda, Grid these days" vol. 1; no. 6; pp. 22-25

2. R.Esposito, P.Mastroserio et al., (March 2003) "wellknown FTP and Grid FTP Protocols for worldwide facts Bypass in Pamela satellite tv for pc place test". [Online]. Available: http://arxiv.Org/ftp/hep-ex/papers/0305/0305084.Pdf

3. Krishna Nadiminti , Srikumar Venugopal et al. "The Gridbus Grid service supplier and Scheduler (v.2.2) man or woman Manual", [Online] to be had: http://www.Cloudbus.Org/dealer/2.2/manualv2.2.Pdf
4. Dorian C. Arnold, Sathish S. Vadhiyar, and Jack Dongarra, At the Convergence of Computational and statistics Grids Parallel Processing Letters, volume eleven, Numbers 2 and three, June and September 2001, pp 187-202. ISSN 01296264.

5. I. Inspire and C. Kesselman. Computational frameworks. In I. Domesticate and C. Kesselman, editors, The Grid: Blueprint for a contemporary Computing Infrastructure. Morgan Kaufmann, 1998

6. Luis Ferreira, Fabiano Lucchese, Tomoari Yasuda, Chin Yau Lee, Carlos Alexandre Queiroz, Elton Minetto, Antonio Mungioli First version (April 2005). Matrix Computing in research and training.

7. http://www.Jatit.Org/explore/introduction_grid_computing. $\mathrm{Htm}$

8. Bart Jacob, Michael Brown, Kentaro Fukui, Nihar Trivedi First version (December 2005). Prologue to Grid Computing. 